\title{
NEW INEQUALITIES FOR $A H$-CONVEX FUNCTIONS USING BETA AND HYPERGEOMETRIC FUNCTIONS
}

\author{
HURIYE KADAKAL ${ }^{\dagger}$ AND KERIM BEKAR
}

\author{
Date of Receiving : $\quad 16.05 .2019$ \\ Date of Revision : $\quad 30.07 .2019$ \\ Date of Acceptance : 11.09 .2019
}

\begin{abstract}
In this manuscript, we obtain some new general inequalities in the form of Hermite-Hadamard type and Bullen type for functions whose third derivatives in absolute value at certain power are arithmetically-harmonically convex via beta and hypergeometric functions. Some applications to special means of real numbers are also given.
\end{abstract}

\section{Introduction}

A function $f: I \subseteq \mathbb{R} \rightarrow \mathbb{R}$ is said to be convex if the inequality

$$
f(t x+(1-t) y) \leq t f(x)+(1-t) f(y)
$$

valid for all $x, y \in I$ and $t \in[0,1]$. If this inequality reverses, then $f$ is said to be concave on interval $I \neq \varnothing$. This definition is well known in the literature.

Convexity theory has appeared as a powerful technique to study a wide class of unrelated problems in pure and applied sciences. Many papers have been written by researchers concerning inequalities for different classes of convex functions see for instance the recent papers $[1,3,4,6,7,8,14,13,16]$ with the other references mentioned therein.

Let $f: I \subseteq \mathbb{R} \rightarrow \mathbb{R}$ be a convex function defined on the interval $I$ of real numbers and $a, b \in I$ with $a<b$. The following inequality

$$
f\left(\frac{a+b}{2}\right) \leq \frac{1}{b-a} \int_{a}^{b} f(x) d x \leq \frac{f(a)+f(b)}{2} .
$$

holds. The inequality (1.1) is known in the literature as Hermite-Hadamard integral inequality for convex functions. Moreover, it is known that some of the classical

2010 Mathematics Subject Classification. 26A51, 26D15.

Key words and phrases. Convex function, $A H$-convex function, beta and hypergeometric functions, Hermite-Hadamard and Bullen type inequality.

Communicated by: Nikhil Khanna

${ }^{\dagger}$ Corresponding author. 